\title{
Comparison the Precision of Ear and Finger Pulse Oximetry with Finger Sensor in Mechanically Ventilated ICU Patients
}

\section{ARTICLE INFO}

\section{Article Type}

Original Research

\section{Authors}

Baloochi Beydokhti T. ${ }^{1} M S c$

Mohammadpour A. ${ }^{1} P h D$

Kianmehr M. ${ }^{2} P h D$,

Shaban M.J.* $M S c$

How to cite this article
Baloochi Beydokhti T, Mohamm-
adpour A, Kianmehr M,Shaban MJ.
Comparison the Precision of Ear
and Finger Pulse Oximetry with
Finger Sensor in Mechanically
Ventilated ICU Patients. Horizon
of Medical Sciences. 2017;23(2)
$: 105-109$.

*Student Research Committee, Gonabad University of Medical Sciences, Gonabad, Iran

${ }^{1}$ Medical-Surgical Nursing Department, Nursing \& Midwifery Faculty, Gonabad University of Medical Sciences, Gonabad, Iran

${ }^{2}$ Medical Physics Department, Medicine Faculty, Gonabad University of Medical Sciences, Gonabad, Iran

\section{Correspondence}

Address: Gonabad University of Medical Sciences, Imam Khomeyni Street, Gonabad, Razavi Khorasan Province, Iran

Phone: +98 (51) 57723115

Fax: -

shabanmj1@mums.ac.ir

\section{Article History}

Received: October 17, 2016

Accepted: January 25, 2016

ePublished: March 25, 2017

\section{A B S T R A C T}

Aims Nowadays, the pulse oximetry is widely used to monitor the oxygen saturation constantly. In the clinical care, it is observed that the ear pulse oximetry with the finger sensor is sometimes utilized in the ICUs. The aim of the study was to determine the accuracy of the ear pulse oximetry and the fingertip with the finger sensor in the ICU patients under the mechanical ventilation.

Materials \& Methods In the analytic comparative study, 60 hospitalized patients under the mechanical ventilation in the ICU of Moddares Hospital in Kashmar Township were studied in 2015. The subjects were selected via available convenience sampling method. One finger pulse oximetry sensor having been attached to the upper part of the ear and the other to the fingertip, the arterial blood sampling was done simultaneously. Data was analyzed by SPSS 22 software using repeated ANOVA and Spearman correlation tests.

Findings Mean oxygen saturation percentage and the heartbeat of ear, fingertip, and arterial blood pulse oximetry were significantly different $(\mathrm{p}<0.001)$. In addition, the oxygen saturation percentage of ear, fingertip, and arterial blood pulse oximetry was significantly correlated by the partial pressure of oxygen (Pa02; $\mathrm{p}=0.001)$. Nevertheless, the mentioned parameters were not significantly correlated by the peripheral edema and especially, by the hand ( $\mathrm{p}>0.05)$.

Conclusion The utilization of ear pulse oximetry with the finger sensor on the fingertip cannot lead to accurate results of the oxygen saturation in the hospitalized patients in the ICU under the mechanical ventilation. However, in case of any reduction in the arterial blood oxygen pressure, the accuracy of the finger pulse oximetry starts to drop out.

Keywords Oximetry; Arterial Oxygen Saturation; Intensive Care Units

\section{CITATION LINKS}

[1] Evaluation of standards in intensive care units in Isfahan Hospitals [2] Determinants of outcome in patients admitted to a surgical intensive care unit [3] Monitoring costs in the ICU: A search for a pertinent methodology [4] How position affects oxygenation: Good lung down? [5] Nurses' perceptions of patient safety climate in intensive care units: A cross-sectional study [6] Evidence for the need of bedside accuracy of pulse oximetry in an intensive care unit [7] Pulse Oximetry. Intensive Care Med [8] Basic nursing: Essentials for practice [9] Delays in the detection of hypoxemia due to site of pulse oximetry probe placement [10] Pulse oximetry [11] Pulse oximeter failure threshold in hypotension and vasoconstriction [12] Accuracy and precision of buccal pulse oximetry [13] The effects of motion artifact and low perfusion on the performance of a new generation of pulse oximeters in volunteers undergoing hypoxemia [14] The effects of motion on the performance of pulse oximeters in volunteers [15] The ear as an alternative site for a pulse oximeter finger clip sensor [16] Validity of pulse oximetry in detection of hypoxemia in children: comparison of ear, thumb and toe probe placements [17] Accuracy of response of six pulse oximeters to profound hypoxia [18] Meta-analysis of arterial oxygen saturation monitoring by pulse oximetry in adults [19] Ayers D, Landry G. Accuracy of pulse oximeters in estimating heart rate at rest and during exercise [20] Pulse oximetry and high-dose vasopressors: A comparison between forehead reflectance and finger transmission sensors [21] The effect of sensor malpositioning on pulse oximeter accuracy during hypoxemia 
امروزه از پالساكسيمترى بهطور كَستردهاى بهمنظور مانيتورينگ

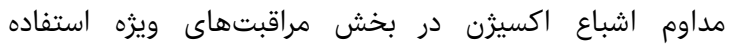

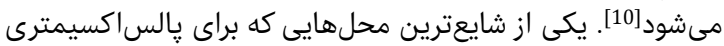

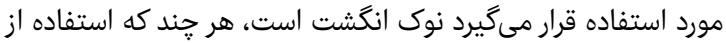

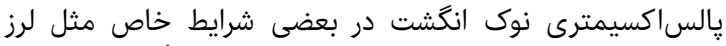

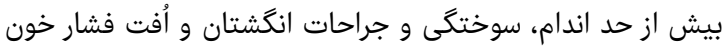

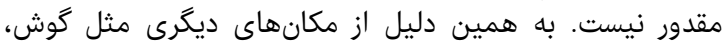

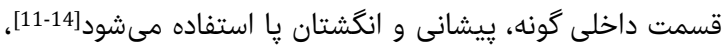

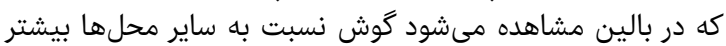

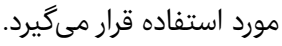

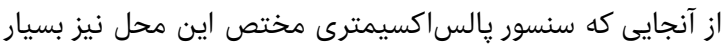

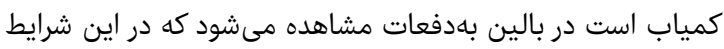

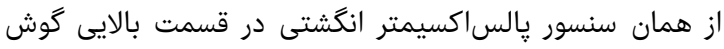

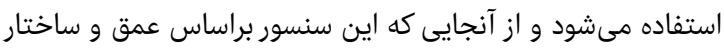

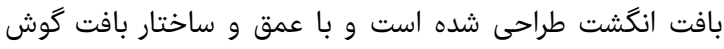

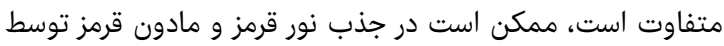

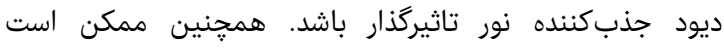

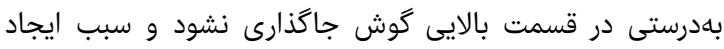

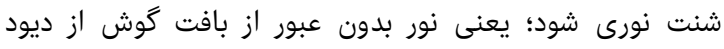

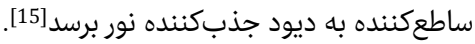

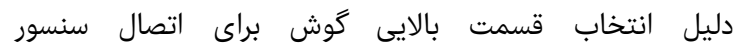

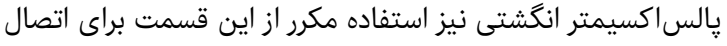

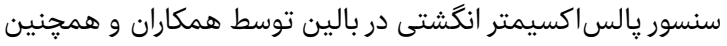

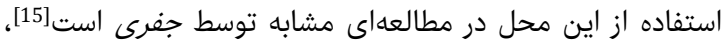

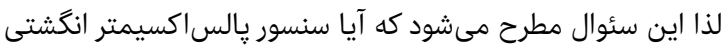

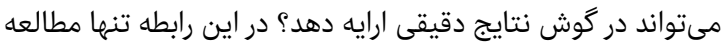

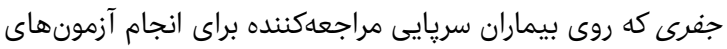

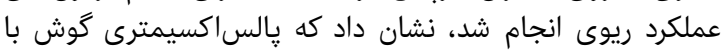

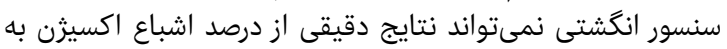

ما ارايه دهد [15]. - مان.

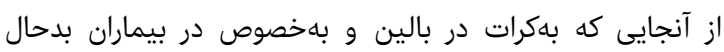

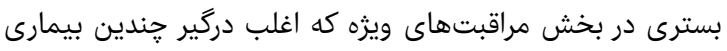

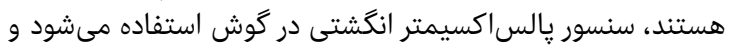

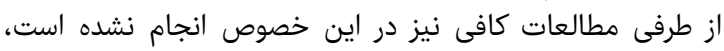

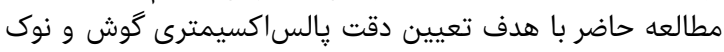

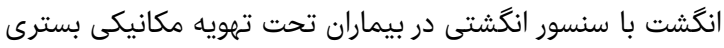

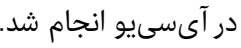

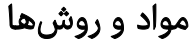

يُزوهش حاضر يك مطالعه تحليلى مقايسهاى است كه در در سال

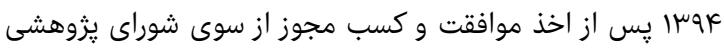

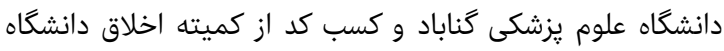

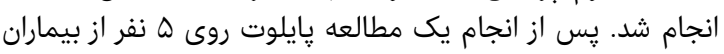

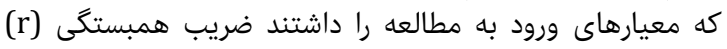

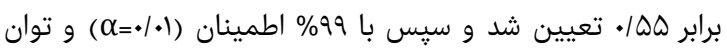

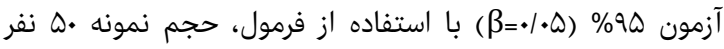

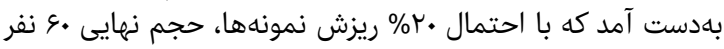
تعيين شد.

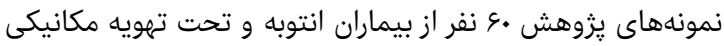

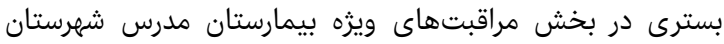

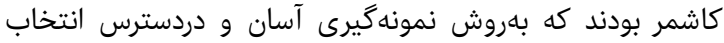

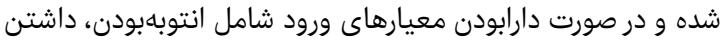

دوره سr، شماره r، بهار
مقايسه دقت يالس اكسيمترى گوش و انگشت بان

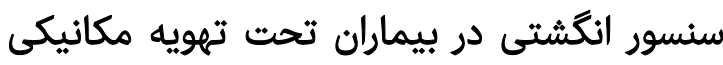
بسترى در بخش مراقبت ويزٔه درو بئه

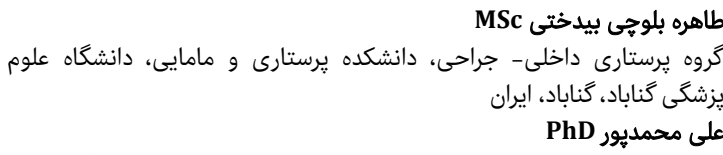

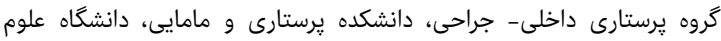

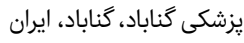

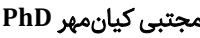

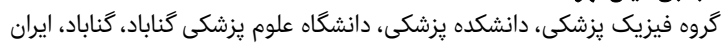

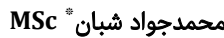

كميته تحقيقات دانشجويى، دانشكاه علوم يزشكى گَناباد، گناباد، ايران

جكيده

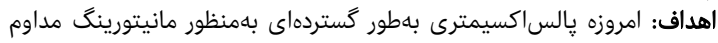

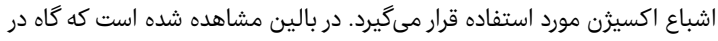

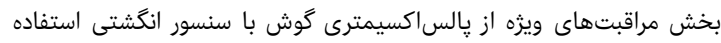

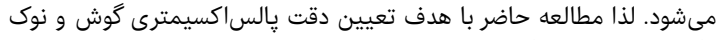
انگشت با سنسور انگشتى در بيماران تحت تهويه مكانيه دانيكى بسترى در بخش مراقبت ويزه انجام شد.

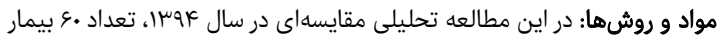

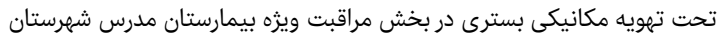

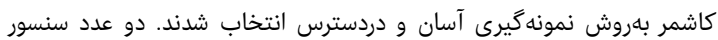

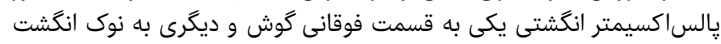

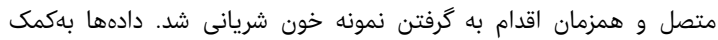

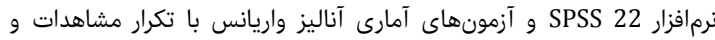

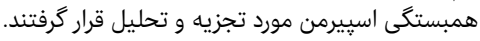

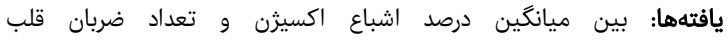

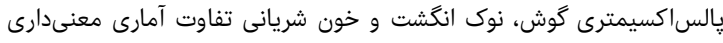

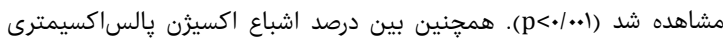

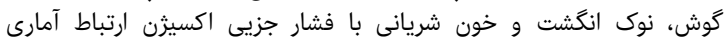

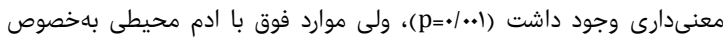

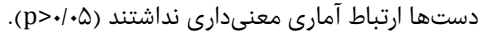

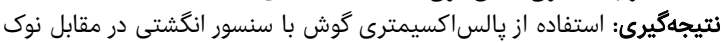

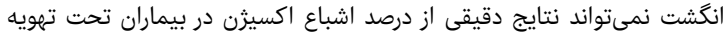

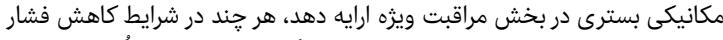

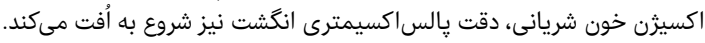

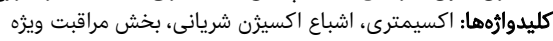

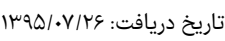

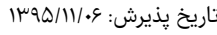

Shabanmj1@mums.ac.ir :نويسنده مسئول:

مقدمه

بخش مراقبتهاى ويزه، بخشى تخصصى و و بسيار يرهزينه در

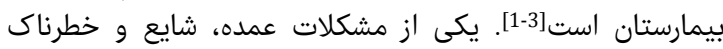

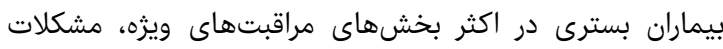

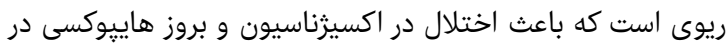

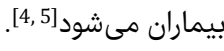

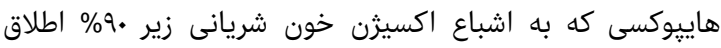

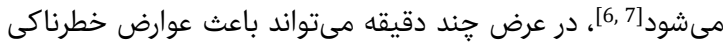

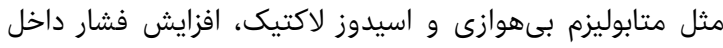

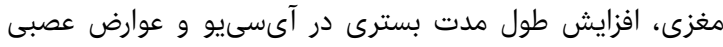

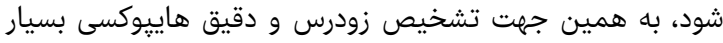
حايز اهميت است [8,

فصلنامه علمى - يزوهشى افق دانش 


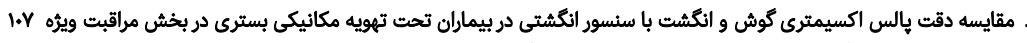

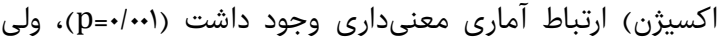

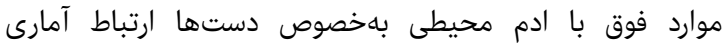

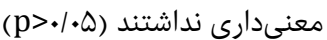

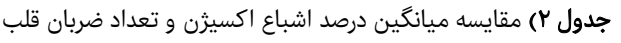

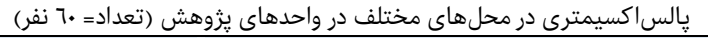
ميانگين متغيرها

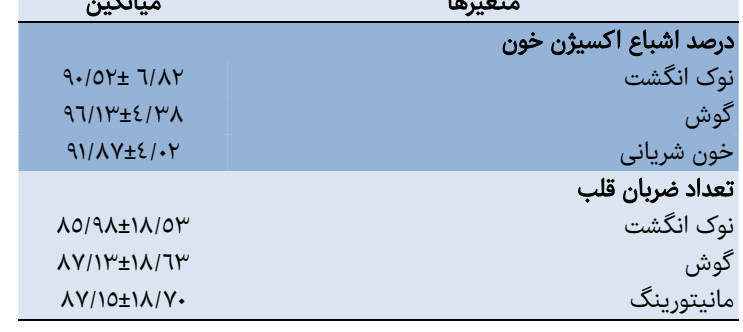

همجنين درصد اشباع اكسيثن در افراد با PaO

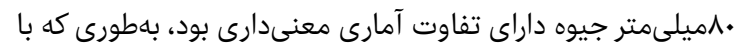

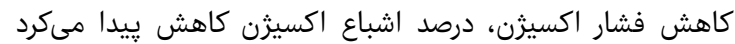

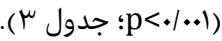

\begin{tabular}{|c|c|c|}
\hline \multicolumn{3}{|c|}{ 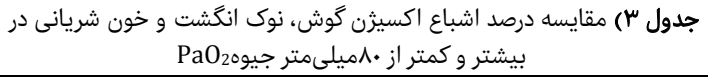 } \\
\hline ميانكين & تعداد & محل \\
\hline & & PaO بيشتر از •^ ميلىمتر جيوه \\
\hline 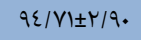 & rı & انگشت \\
\hline $99 / 1 \cdot \pm \cdot 199$ & rᄉ & كوش \\
\hline $9 \varepsilon / 0 \wedge \pm r / / V$ & rᄉ & خون شريانى \\
\hline & & PaO $\mathrm{PaO}_{2}$ \\
\hline$\Lambda \mathrm{T} / \Lambda \varepsilon \pm V / 10$ & Hr & انگشت \\
\hline $9 \Psi / 0 \mu \pm \varepsilon / 00$ & rr & كوش \\
\hline$\Lambda 9 / 0 . \pm \mu / \vee 0$ & rr & خون شريانى \\
\hline
\end{tabular}

ميانكين اختلاف درصد اشباع اكسيثن نوك انگشت با خون خون

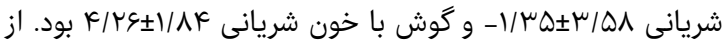

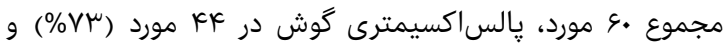

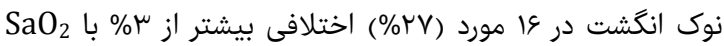
داشت.

بحث

در اين يزوهش با توجه به دادهها، پيالساكسيمترى گوش در

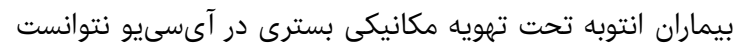

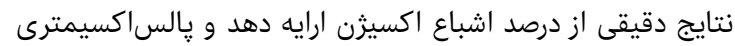

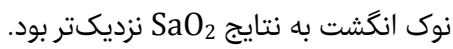

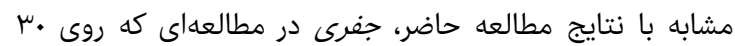

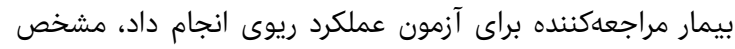

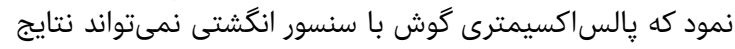
قابل قبولى از درصد اشباع اكسيثن ارايه دهد و

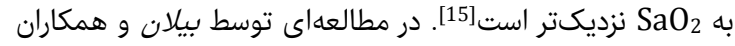

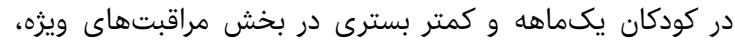

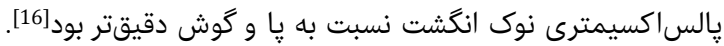

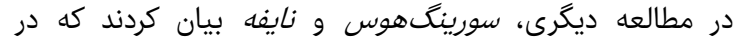

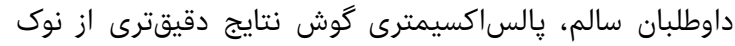

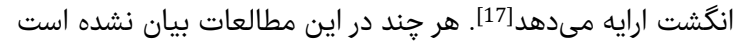

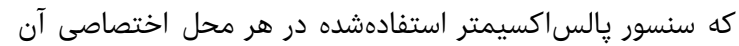

دستور يزشك براى ABG (كازهاى خون شريانى) و عدم سوختگى

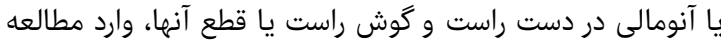

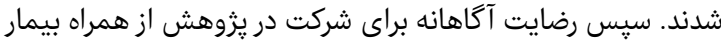

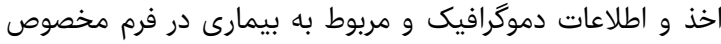

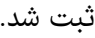

از بيماران تحت مطالعه يكميلىليتر خون شريانى داخل سرنگ

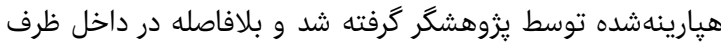

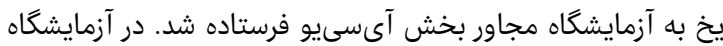

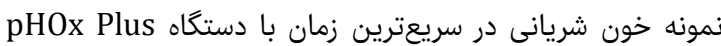
Nova Biomedical)

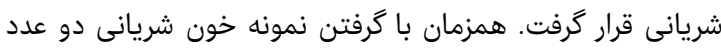

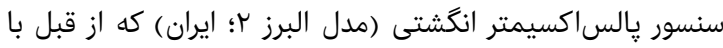

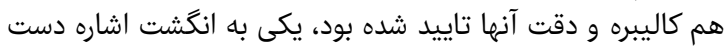

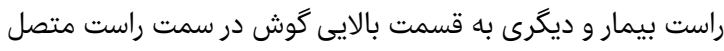
شد. SpO

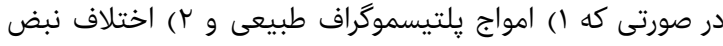

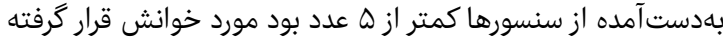

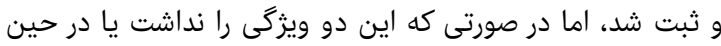

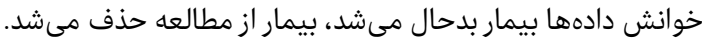
سيس نتايج بهدستآمده از

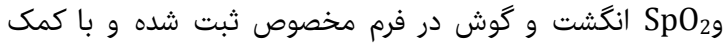

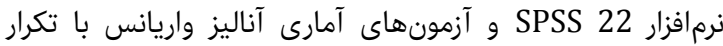

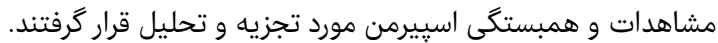

يافتهها

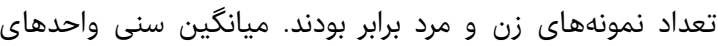

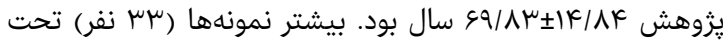

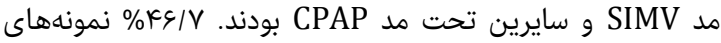
يُزوهش ادم محيطى داشتند (جدول ().

جدول () توزيع فراوانى ويزگگهاى افراد تحت مطالعه

\begin{tabular}{|c|c|c|}
\hline درصد & تعداد & متغيرها \\
\hline & & جنسيت \\
\hline Q. & $\mu$. & زن ن من \\
\hline \multirow[t]{2}{*}{$\omega \cdot$} & $\mu$. & مرد | مرد \\
\hline & & مد دستگًاه تهويه \\
\hline$K Q$ & TV & مد CPAP \\
\hline \multirow[t]{2}{*}{$\Delta \Delta$} & سر & SIMV \\
\hline & & ادم محيطى \\
\hline$\Delta \mu / \mu$ & rr & ندارد \\
\hline 10 & 9 & +1 \\
\hline · & · & $+r$ \\
\hline$r$. & $\pi$ & $+\mu$ \\
\hline $11 / V$ & v & $+k$ \\
\hline
\end{tabular}

بين ميانكين درصد اشباع اكسيزن پالساكسيمترى گوش، نوى

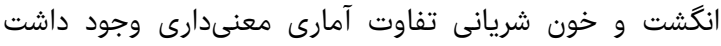

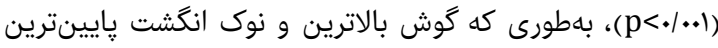

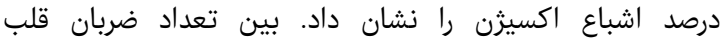

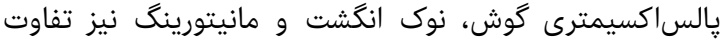

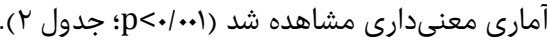

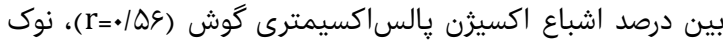

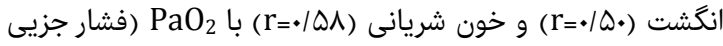


ييشنهاد مى شود مطالعهاى به منظور بررسى دقت يالساكسيمترى كوش با سنسور ويزه در بخش مراقبتهاى مئ ويزه انجام شود.

\section{نتيجه}

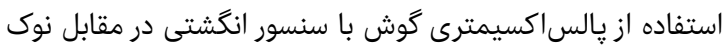

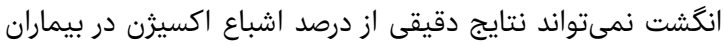

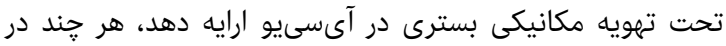

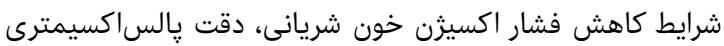

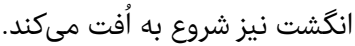

تشكر و قدردانى: در يايان از استاد محترم جناب آقاى مهدى

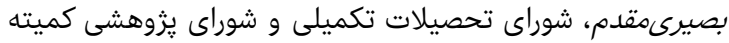

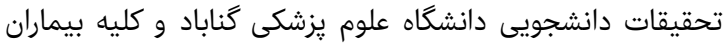

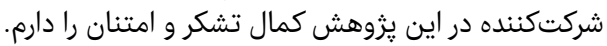

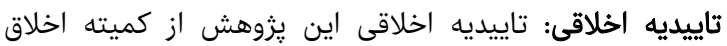

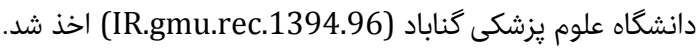
تعارض منافع: هيج موردى توسط نويسندگًان گزارش نارش نشده است.

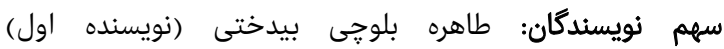

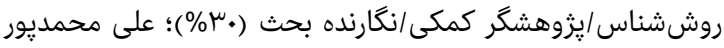

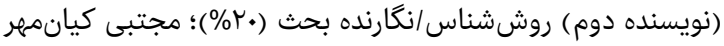

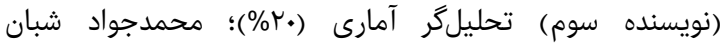

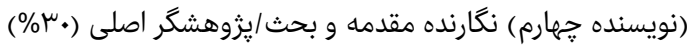

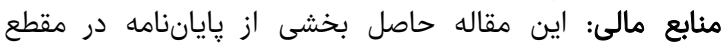

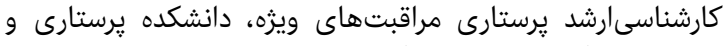
مامايى دانشگاه علوم يزشكى گَناباد است.

\section{منابع}

1- Ayoubian A, Moazam E, Navid M, Hoseinpourfard M, Izadi M. Evaluation of standards in intensive care units in Isfahan Hospitals, Iran. J Mil Med. 2013;14(4):255-62. [Persian]

2- Abelha F, Maia P, Landeiro N, Neves A, Barros H. Determinants of outcome in patients admitted to a surgical intensive care unit. Arq Med. 2007;21(5-6):13543.

3- Reis Miranda D, Jegres M. Monitoring costs in the ICU: A search for a pertinent methodology. Acta Anaesthesiol Scand. 2012;56(9):1104-13.

4- Yeaw EM. How position affects oxygenation: Good lung down?. Am J Nurs. 1992;92(3):26-9.

5- Ballangrud R, Hedelin B, Hall-Lord ML. Nurses' perceptions of patient safety climate in intensive care units: A cross-sectional study. Intensive Crit Care Nurs. 2012;28(6):344-54.

6- Seguin P, Le Rouzo A, Tanguy M, Guillou YM, Feuillu A, Mallédant Y. Evidence for the need of bedside accuracy of pulse oximetry in an intensive care unit. Crit Care Med. 2000;28(3):703-6.

7- Jubran A. Pulse 0ximetry. Intensive Care Med. 2004;30(11):2017-20.

8- Potter PA, Perry AG. Basic nursing: Essentials for practice. $5^{\text {th }}$ edition. Philadelphia: Mosby; 2007.

9- Hamber EA, Bailey PL, James SW, Wells DT, Lu JK, Pace NL. Delays in the detection of hypoxemia due to site of pulse oximetry probe placement. J Clin Anesth. 1999;11(2):113-8.

10- Jubran A. Pulse oximetry. Crit Care. 2015;19(1):272-6.

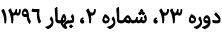

1•ا طاهره بلوجى بيدختى و همكاران

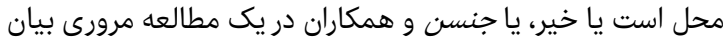

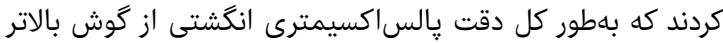

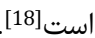

در مطالعه حاضر يالساكسيمترى گوش نسبت به انگشت تعداد

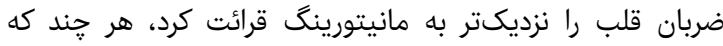

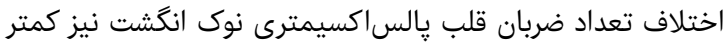

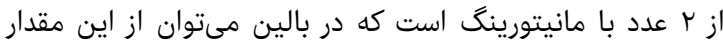

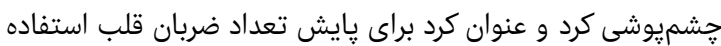

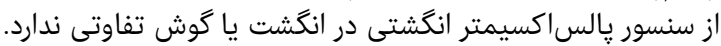

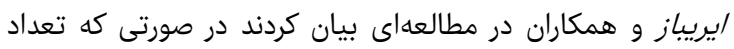

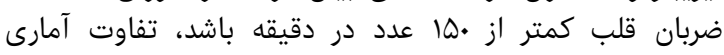

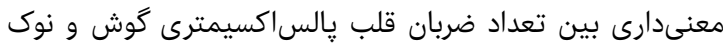
انكشت با مانيتورينگ وجود بين تعداد فربان ندارد [19].

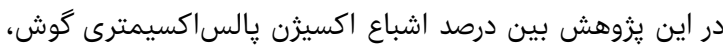

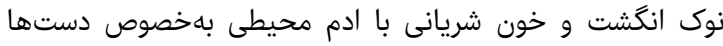

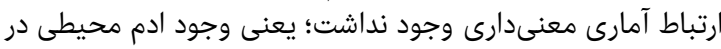

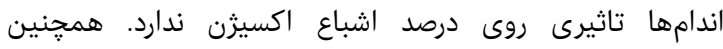

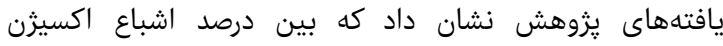

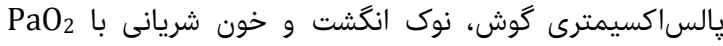

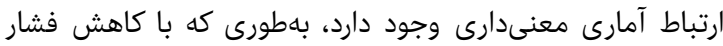

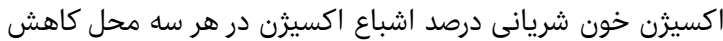
ييدا مىكرد، ولى

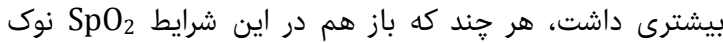
انكُشت به

انگشت از گوش دقيقتر است.

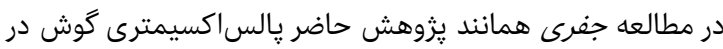

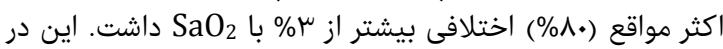

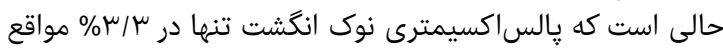

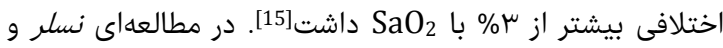

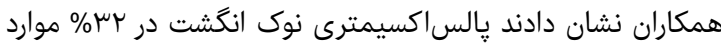

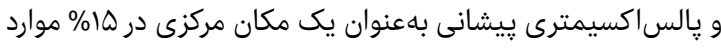

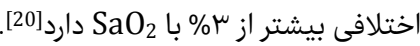

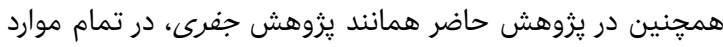

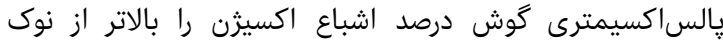

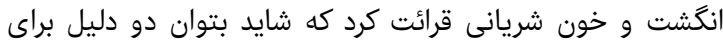

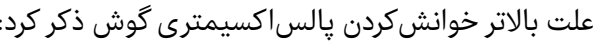

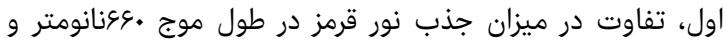

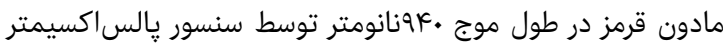

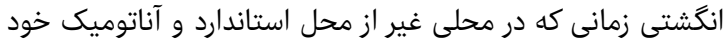

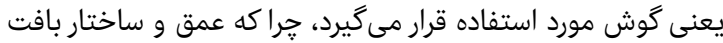

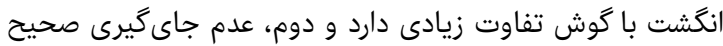

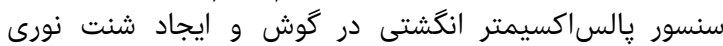

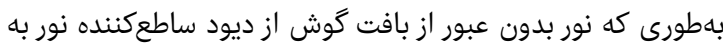

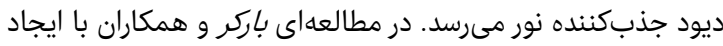

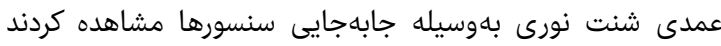

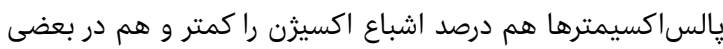

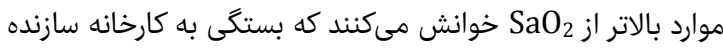
و مدل يالساكسيمتر و ميزان

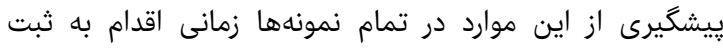

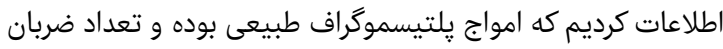

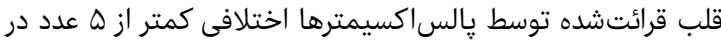

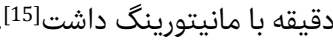

فصلنامه علمى- هيروهشى افق دانش 
مقايسه دقت يالس اكسيمترى گوش و انكشت با سنسور انكشتى در بيماران تحت تهويه مكانيكى بسترى در بخش مراقبت ويره 1.9

comparison of ear, thumb and toe probe placements.

East Mediterr Health J. 2010;16(2):218-22.

17- Severinghaus JW, Naifeh KH. Accuracy of response of six pulse oximeters to profound hypoxia. Anesthesiology. 1987;67(4):551-8.

18- Jensen AL, Onyskiw JE, Prasad NG. Meta-analysis of arterial oxygen saturation monitoring by pulse oximetry in adults. Heart lung. 1998;27(6):387-408.

19- Iyriboz Y, Powers S, Morrow J, Ayers D, Landry G. Accuracy of pulse oximeters in estimating heart rate at rest and during exercise. $\mathrm{Br} J$ Sports Med. 1991;25(3):162-64.

20- Nesseler N, Frénel JV, Launey Y, Morcet J, Mallédant Y, Seguin P. Pulse oximetry and high-dose vasopressors: A comparison between forehead reflectance and finger transmission sensors. Intensive Care Med. 2012;38(10):1718-22.

21- Barker SJ, Hyatt J, Shah NK, Kao YJ. The effect of sensor malpositioning on pulse oximeter accuracy during hypoxemia. Anesthesiology. 1993;79(2):248-54.
11- Severinghaus JW, Spellman MJ Jr. Pulse oximeter failure threshold in hypotension and vasoconstriction. Anesthesiology. 1990;73(3):532-7.

12- De Jong MJ, Schmelz J, Evers K, Bradshaw $\mathrm{P}$ McKnight K, Bridges E. Accuracy and precision of buccal pulse oximetry. Heart Lung. 2011;40(1):31-40.

13- Gehring H, Hornberger C, Matz H, Konecny E, Schmucker $\mathrm{P}$. The effects of motion artifact and low perfusion on the performance of a new generation of pulse oximeters in volunteers undergoing hypoxemia. Respir Care. 2002;47(1):48-60.

14- Barker SJ, Shah NK. The effects of motion on the performance of pulse oximeters in volunteers (revised publication). Anesthesiology. 1997;86(1):101-8.

15- Haynes JM. The ear as an alternative site for a pulse oximeter finger clip sensor. Respir Care. 2007;52(6):727-9.

16- Bilan N, Behbahan AG, Abdinia B, Mahallei M. Validity of pulse oximetry in detection of hypoxemia in children: 